\title{
Research Paper \\ The Effects of L-dopa, SCH23390 Hydrochloride and Sulpiride on Adiponectin and Luteinizing Hormone Levels in an Animal Model of Polycystic Ovary Syndrome
}

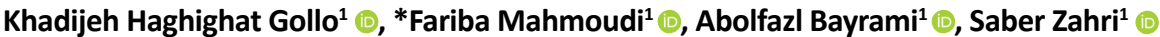

1. Department of Biology, Faculty of Sciences, University of Mohaghegh Ardabili, Ardabil, Iran.

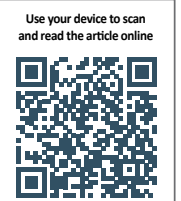

Crtation: Haghighat Gollo Kh, Mahmoudi F, Bayrami A, Zahri S. [The Effects of L-dopa, SCH23390 Hydrochloride and Sulpiride on Adiponectin and Luteinizing Hormone Levels in an Animal Model of Polycystic Ovary Syndrome (Persian)]. Journal of Arak University of Medical Sciences (JAMS). 2020; 23(2):162-171. https://doi.org/10.32598/JAMS.23.2.5015.1

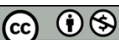

Article Info:

Received: 03 Dec 2019

Accepted: 18 Feb 2020 Available Online: 01 Jun 2020

Key words:

L-dopa, Adiponectin, Polycystic ovary syndrome, Sulpiride, $\mathrm{SCH} 23390$

\begin{abstract}
Background and Aim In patients suffer from Polycystic Ovary Syndrome (PCOS), the secretion of the Luteinizing Hormone ( $\mathrm{LH}$ ) increases while adiponectin secretion and dopamine release decreases. Dopamine and adiponectin exert inhibitory effecs on LH secretion. In the present study the effects of L-dopa and dopamine receptor antagonists were investigated on $\mathrm{LH}$ secretion and adiponectin gene expression of in PCOS model rats to determine whether dopaminergic pathway might be involved in the decreasing LH via affecting adiponectin.

Methods \& Materials Following estradiol valerate- induced PCOS, fifteen PCOS rats were divided into 3 groups including saline receiving group, L-dopa $(100 \mathrm{mg} / \mathrm{kg})$ or simultaneous injections of sulpride(10 mg/ $\mathrm{kg}), \mathrm{SCH} 23390$ hydrochloride $(1 \mathrm{mg} / \mathrm{kg})$ and L-dopa(100 mg/kg), Five intact rats received saline as negative control group. Blood samples were collected via tail vein. Ovary and hypothalamus were dissected and frozen. Serum concentration of LH and relative gene expression of adiponectin in ovary and hypothalamus were determined by radioimmunoassay and real time-PCR method.

Ethical Considerations This study was approved by the Research Committee of University of Mohaghegh Ardabili (Code: 95.125.1)

Results Induction of PCOS caused a significant increase in mean serum concentration of LH and a significant decrease in mean relative gene expression of ovarian and hypothalamic adiponectin compared to control group. L-dopa caused a significant decrease in serum concentration of $\mathrm{LH}$, a significant decrease in hypothalamic gene expression of adiponectin compared to PCOS rats. But it did not significantly increase ovarian adiponectin gene expression in comparison to PCOS rats. Dopamine receptor antagonists inhibit the effects of L-dopa on LH and hypothalamic gene expression of adiponectin.

Conclusion Dopaminergic signaling pathway may be involved in decreasing LH secretion via increasing hypothalamic adiponectin gene expression level in PCOS rats.
\end{abstract}

\footnotetext{
Extended Abstract

Introduction

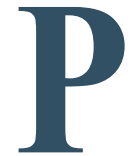

olycystic Ovary Syndrome (PCOS) is associated with insulin resistance, elevated serum androgen levels, and an increase in the ratio of Luteinizing Hormone (LH) to Follicle-

Stimulating Hormone (FSH) $[1,2]$. Adiponectin is synthesized in adipose tissue, hypothalamus, and gonads [3, 4]. Deficiency in adiponectin production leads to insulin resistance and disruption in lipid and glucose metabolisms [7, 8]. Serum adiponectin levels in PCOS women are lower than in healthy individuals $[8,10]$. L-dopa is a precursor to the neurotransmitters dopamine, epinephrine and norepi-

* Corresponding Author

Fariba Mahmoud, PhD.

Address: Department of Biology, Faculty of Sciences, University of Mohaghegh Ardabili, Ardabil, Iran.

Tel: +98 (914) 4190422

E-mail: f.mahmoudi@uma.ac.ir
} 
nephrine [14]. Dopamine and L-dopa inhibit Hypothalamic-Pituitary-Gonadal (HPG) axis activity $[15,16]$. PCOS is associated with decreased dopamine release [19]. This study aimed to examine the effects of L-dopa and dopamine receptor antagonists (SCH 23390 as D1 receptor and sulperide as D2 receptor) on LH secretion and relative expression of adiponectin gene in the hypothalamus and ovaries of rats with PCOS induced by

\section{Materials and Methods}

To perform this study, 20 Wistar female rats weighing 180-220 g were used. In order to induce PCOS, the animals received intramuscular injection of Estradiol Valerate (EV) in the estrous stage. 15 PCOS rats were divided into three groups of saline, L-dopa $(100 \mathrm{mg} / \mathrm{kg})$, and L-dopa + sulpiride + SCH 23390 (100 mg/kg L-dopa + 10mg/ $\mathrm{kg}$ sulpiride $+1 \mathrm{mg} / \mathrm{kg} \mathrm{SCH} 23390$ hydrochloride), and 5 healthy rats received saline as negative control group. In groups receiving antagonist and L-dopa, antagonists were injected 10 minutes before L-dopa injection. The hypo- thalamus and ovarian samples were isolated and stored at $-80^{\circ} \mathrm{C}$ until RNA extraction. The average serum LH concentration was measured using Radioimmunoassay (RIA). The mean relative expression of adiponectin gene in the ovaries and hypothalamus was calculated using real-time PCR assay and delta-delta CT method ( $2^{-\Delta \Delta C t}$ formula). The data obtained from this formula were analyzed in SPSS V. 16 software using one-way ANOVA test and the mean data were compared by using Tukey's post-hoc test. The results were presented as Mean $\pm \mathrm{SD}$, considering the significance level of $\mathrm{P} \leq 0.05$.

\section{Results}

The results of comparing the mean serum LH concentrations in the negative control and PCOS groups are shown in Table 1. The mean relative expression of adiponectin gene in the ovaries and hypothalamus of the PCOS control group showed a significant decrease compared to the negative control group $(\mathrm{P}=0.001$ for ovaries and $\mathrm{P}=0.015$ for hypothalamus) (Figure 1). For the PCOS group received L-do-

Table 1. Comparing the mean serum LH concentrations in the negative control and PCOS groups

\begin{tabular}{|c|c|}
\hline Groups & $\mathrm{ng} / \mathrm{ml}$ \\
\hline Negative control & $2.9 \pm 0.14$ \\
\hline PCOS control & $5.08 \pm 0.11 *(P<0.001)$ \\
\hline L-dopa & $3.18 \pm 0.26 * *(P<0.001)$ \\
\hline L-dopa + sulpiride + SCH23390 & $3.95 \pm 0.14 *(\mathrm{P}=0.003), * *(\mathrm{P}=0.002),{ }^{* * *}(\mathrm{P}=0.029)$ \\
\hline $\begin{array}{l}\text { * Compared to the negative control group; } \\
\text { ** compared to the PCOS control group; }\end{array}$ & SO. Journal of \\
\hline
\end{tabular}

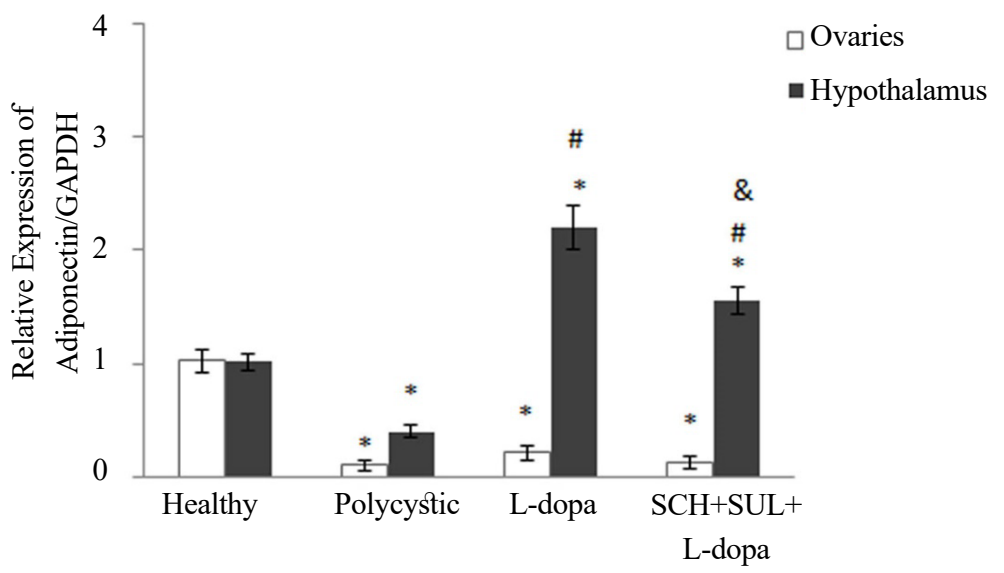

Journal of
Arak University of Medical Sciences

Figure 1. The mean relative expression of adiponectin gene in the ovaries and hypothalamus of rats received saline, L-dopa only, and L-dopa plus sulpiride (SUL) and SCH 23390. * Compared to the healthy group; \# compared to the PCOS control; \&compared to the L-dopa group 
pa only, the mean relative expression of adiponectin gene in the ovaries and hypothalamus increased non-significantly $(\mathrm{P}=0.924)$ and significantly $(\mathrm{P}<0.001)$, respectively (Figure 1). For the PCOS group received L-dopa, sulpiride, and SCH 23390 hydrochloride simultaneously, the mean relative expression of adiponectin gene in the ovaries and hypothalamus decreased non-significantly $(\mathrm{P}=0.948)$ and significantly $(\mathrm{P}=0.025)$, respectively (Figure 1$)$.

Discussion

The results of the present study showed that in PCOS rats, serum LH concentrations increased significantly compared to the healthy rats, while the mean relative expression of adiponectin gene in the ovaries and hypothalamus of PCOS rats reduced significantly compared to the healthy rats. The results are consistent with previous research on the extent of adiponectin secretion in humans and rodents with PCOS. Previous studies have shown that the serum adiponectin level is reduced in women with PCOS compared to healthy peers, and PCOS obese women have lower serum levels than non-PICOS obese women $[8,22]$. Decreased adiponectin levels in PCOS women may be due to increased production of androgens caused by reduced inhibitory effects of adiponectin on theca cells [23], because hyperandrogenism and obesity have been shown to play an important role in reducing plasma levels of adiponectin and causing insulin resistance, which is a major feature of PCOS [24, 25].

L-dopa exerted inhibitory effects on LH secretion and stimulatory effects on adiponectin gene expression in the hypothalamus of PCOS rats; however, it had no stimulatory effects on adiponectin gene expression in the ovaries of PCOS rats. This is consistent with the results of an invitro study where researchers examined the effects of dopamine on adipocyte cells incubated in culture, and reported the stimulatory effects of dopamine on adiponectin secretion from these cells [22]. Dopamine receptor antagonists including SCH23390 hydrochloride and sulpiride blocked the inhibitory effects of L-dopa on the LH secretion and its stimulatory effects on the relative expression of the adiponectin gene in the hypothalamus of PCOS rats. Increasing the activity of dopaminergic neurons may be effective in controlling endocrine disorders caused by decreased adiponectin secretion in PCOS patients.

Ethical Considerations

\section{Compliance with ethical guidelines}

This study ethically approved in ethics committee of University of Mohaghegh Ardabili (Code: 95-125-1).

\section{Funding}

The present paper was extracted from the MSc thesis of the first author, Department of Biology, Faculty of Sciences, University of Mohaghegh Ardabili.

Authors' contributions

All authors contributed in preparing this article.

\section{Conflicts of interest}

The authors declared no conflict of interest.

\section{Acknowledgements}

The authors would like to thank Deputy of Research and Technology of the Mohaghegh Ardabili University for their financial and non-financial supports. Also thank to Dr. Homayoun Khaz'ali from Shahid Beheshti University for providing the instruments. 


\title{
اثرات الدويا، SCH23390 هيدروكلرايد و سوليريد بر آديبونكتين و هورمون لوتئينهكنتده در مدل حيوانى در سندروم تخمدان يلى كيستيك
}

\author{
خديجه حقيقت كللو' ه. "فريبا محمودى' ـ. ابوالفضل بايرامى' (ن. صابر زهرى' (1)
}

1. أكروه زيستشناسى، دانشكده علوم، دانشَّاه محقق اردبيلى، اردبيل، ايران.

\begin{abstract}
هيكيد

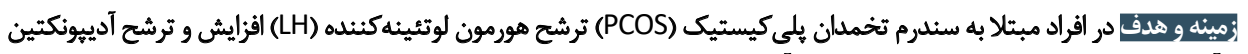

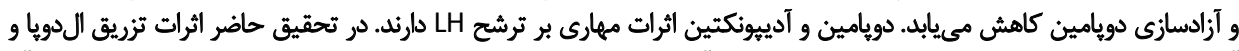

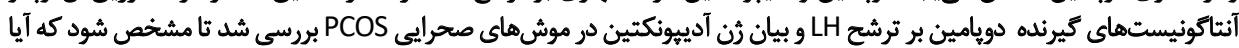

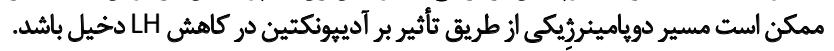

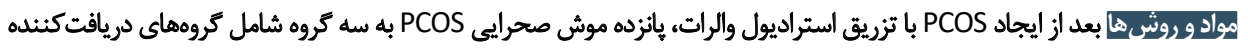

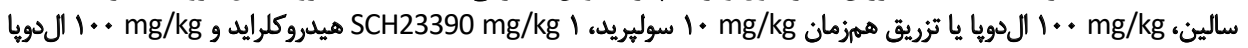

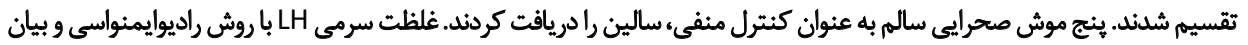

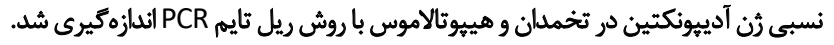

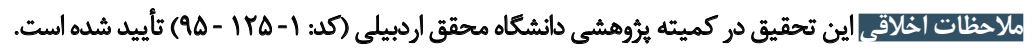

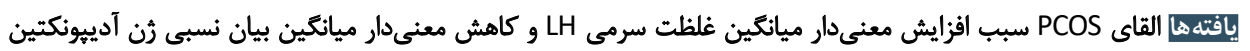

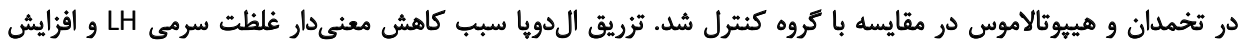

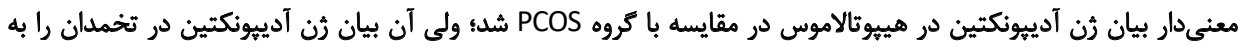

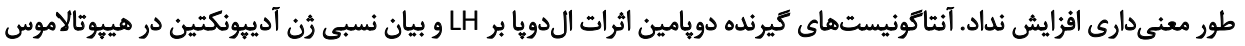
را مهار كردند.

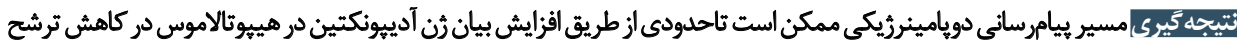

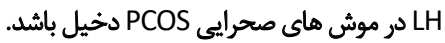

اطلاعات مقاله:

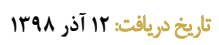
تاريخ هذيرش:

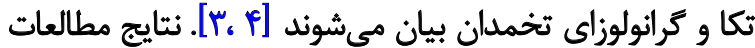

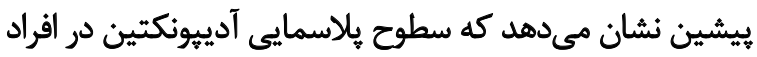

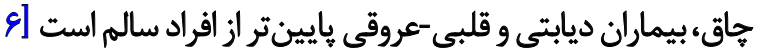

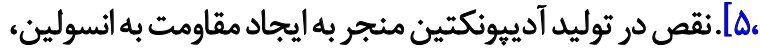

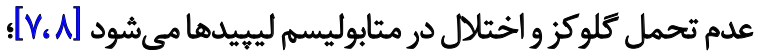

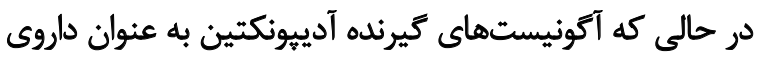

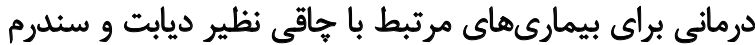

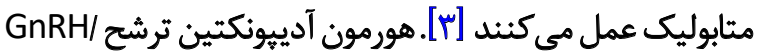

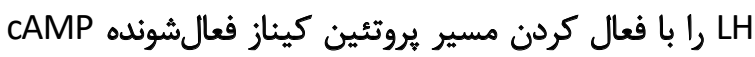
(AMPK)

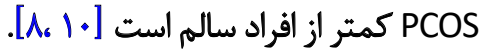

الدويا بيشساز نوروترانسميترهاى دويامين، ايىنفرين و

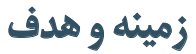

سندروم تخمدان بلى كيستيك (PCOS) يكى از مهمثترين

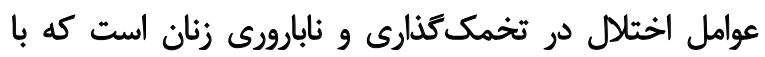

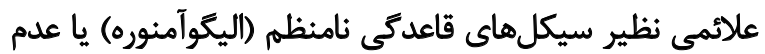

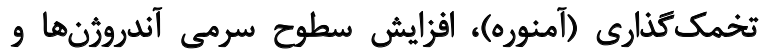

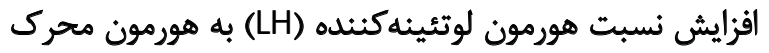

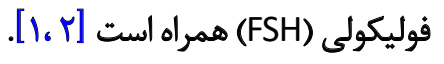

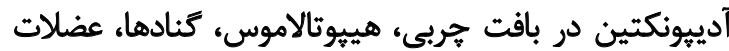

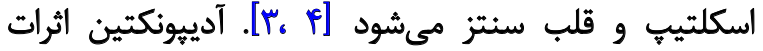

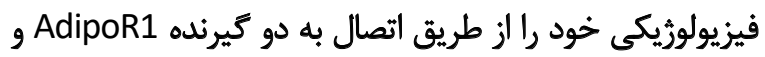

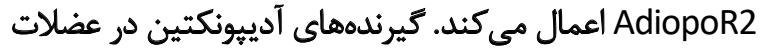

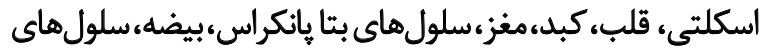

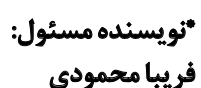

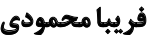
نشاني: اردبيل، دانشُكاه محقق اردبيلي، دانشكده علوم، كروه زيستشناسى.

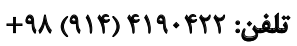
f.mahmoudi@uma.ac.ir يست الكترونيكي 
شدند. براى القاى يلى يلى إيستيك، تزريق عضلانى استر اديول والرات

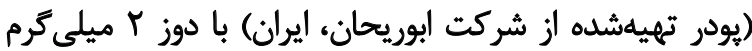

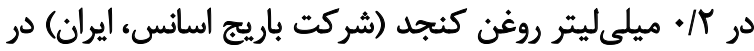

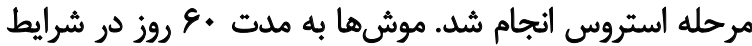

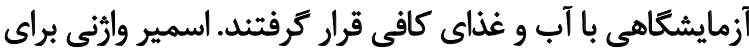

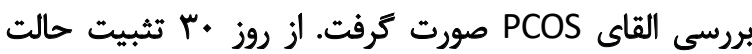

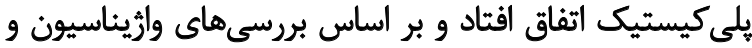

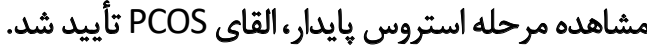

تزريق داروها: براي انجام اين آزمايش ها موش صحرايي PCOS

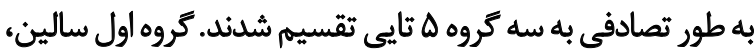

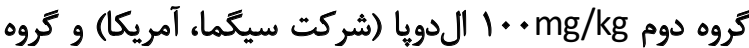

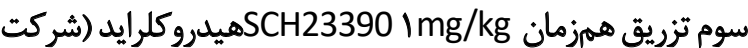

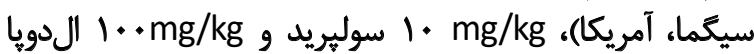

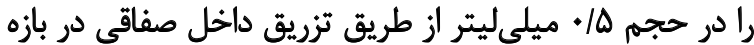

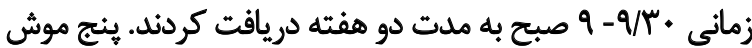

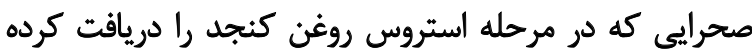

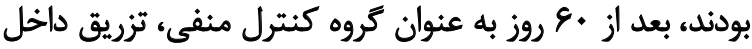

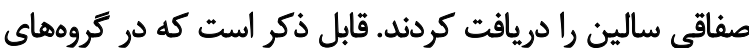
1.

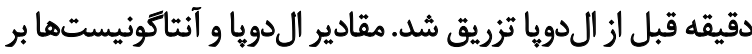

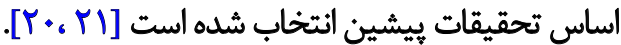

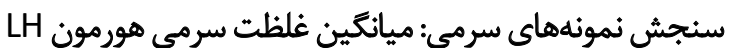

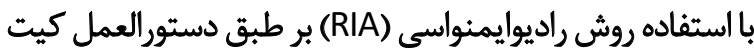

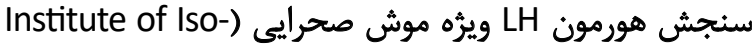
اندازمكيرى شد.

جداسازى نمونههاي بافتى: حيوانات با استفاده از كتامين (A · mg/Kg BW)

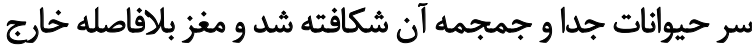

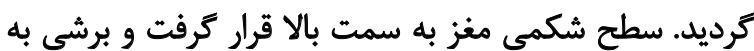

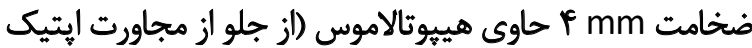

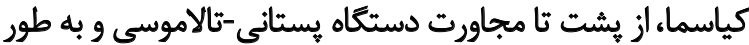

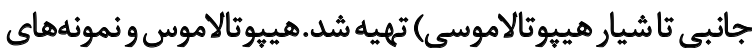

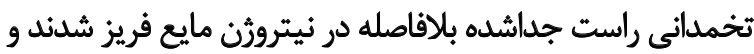

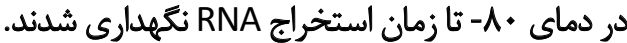

بررسى ميزان بيان رُنى با استفاده از روش ريل ثايم-PCR:

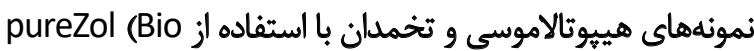
Rad Co, U.S.A) مطلق نمونهها با استفاده از كلروفرم (Merck Co, Germany)،

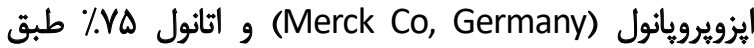
دستورالعمل كيت pureZol (Bio Rad Co, U.S.A) استخرل التواج

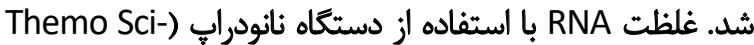

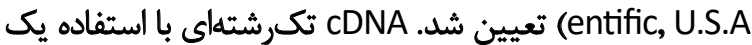

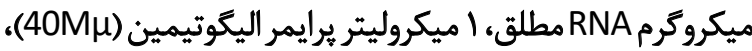

نورايىنفرين است كه از اسيدآمينه L- Lيروزين توسط تيروزين هيدروكسيلاز ايجاد مىشود. نامهاى تجارى آن - pharmaco

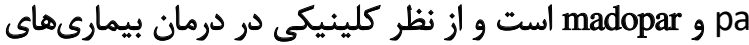

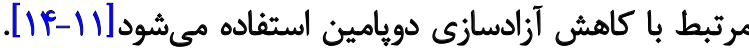

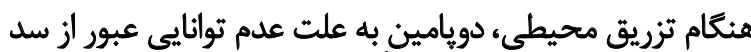

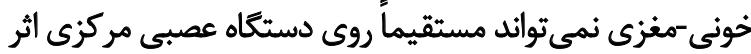

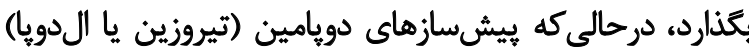

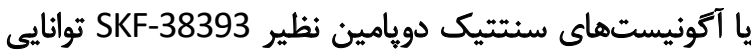

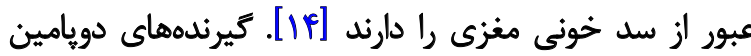

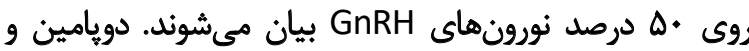

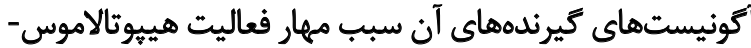
هيبوفيز- كنادها (HPG) و كاهش آزادسازى

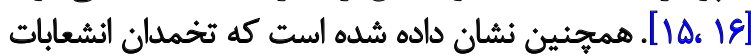

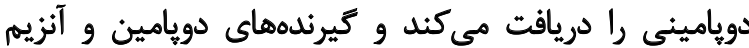

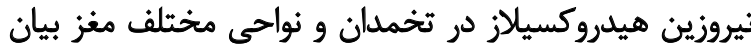

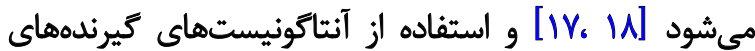

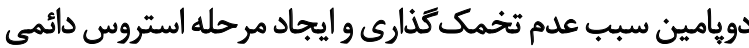

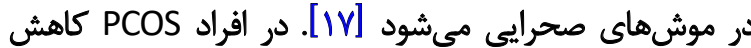

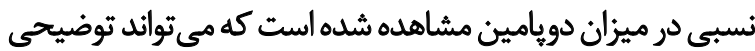

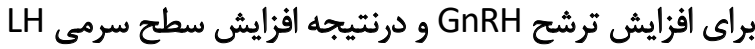

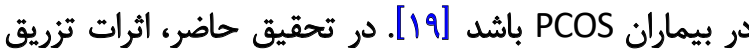

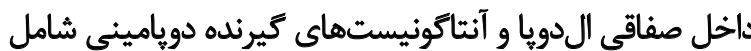

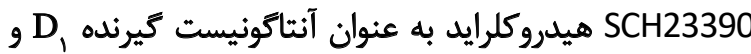

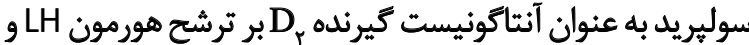

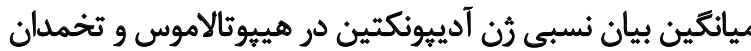

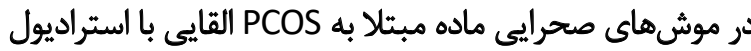

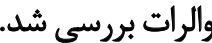
مواد ورشنها واحدهاى آزمايشى: تخقيق حاضر از نوع تجربى بنيادى است.

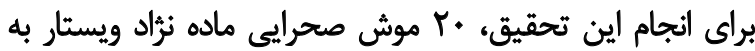

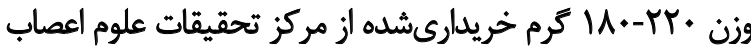

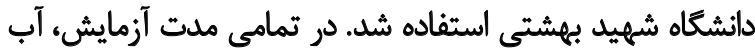

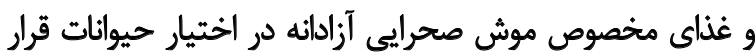

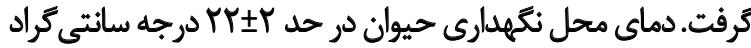

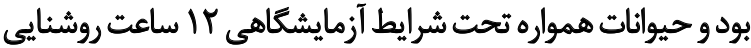

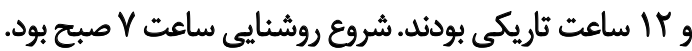
بررسى وازيناسيون و القاى سندروم تخمدان يلى كيستيك:ابتدا

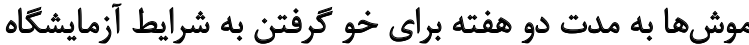

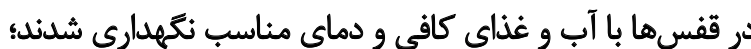

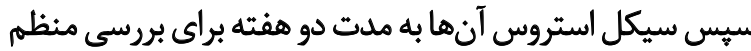

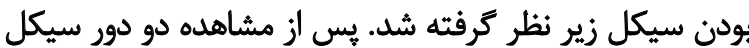

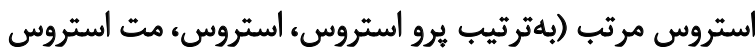
و دى استروس) موشهاى صحرايى براى شروع آزمايش آماده استروس 
ثنايج

نتايج اثرات تزريق سالين، الدويا يا تزريق همزمان

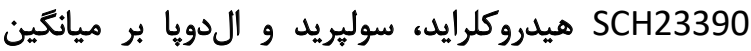

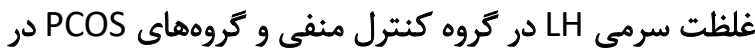
جدول شماره ا خلاصه شده است.

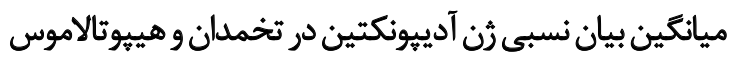

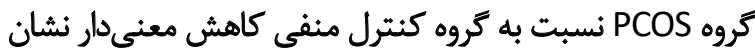

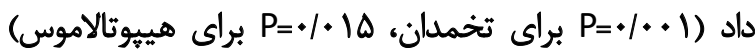

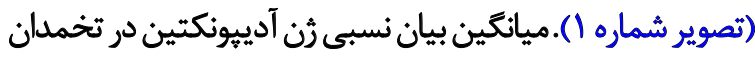

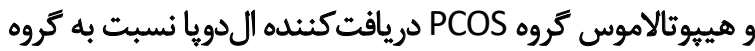
بCOS

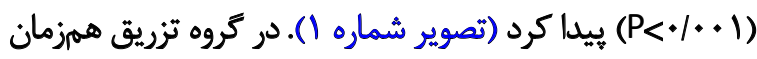

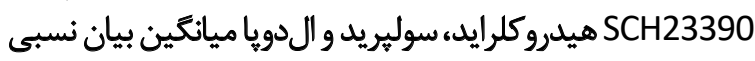

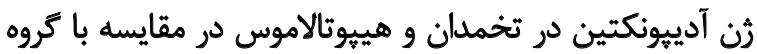

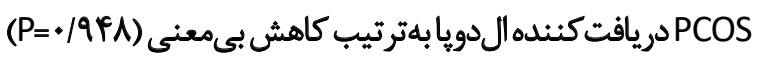

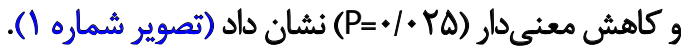

بحث

نتايج حاصل از اين تحقيق نشان داد كه در موشهاي صحرايى

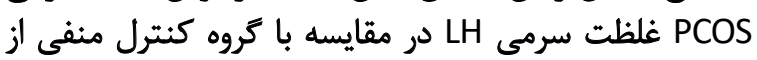

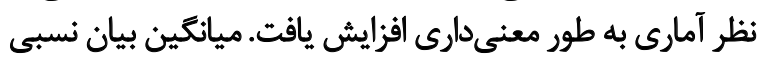

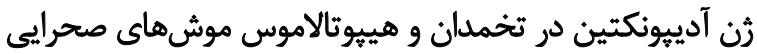

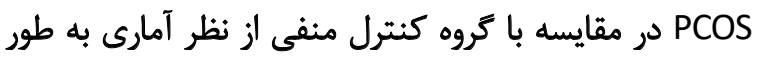

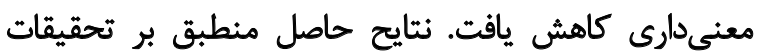

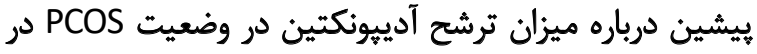

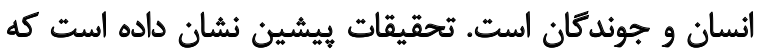

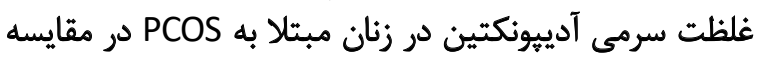

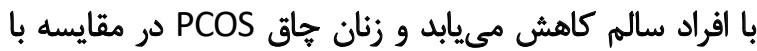

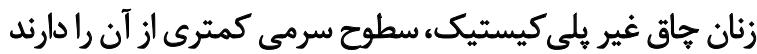

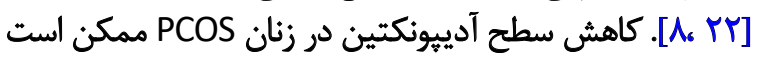

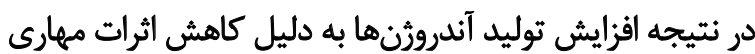

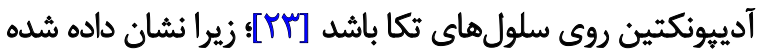

ا ميكروليتر مخلوط نوكلئوتيد ترى فسفاتها (10mMdNTP)،

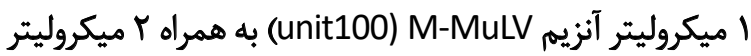

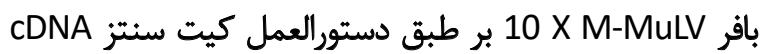
با استفاده از دستًاه ترمال سايكلر (Vivantis Co., Malaysia) سنتز شد. تعيين سطح mRNA سكليسر آلدئيد

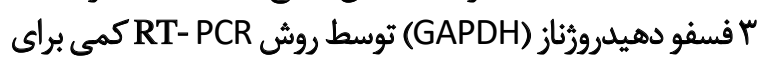
نرمال كردن نمونههاى mRNA آدييونكتين استفاده شد. توري

يس از سنتز cDNA قطعات موردنظر ثنها بر حسب

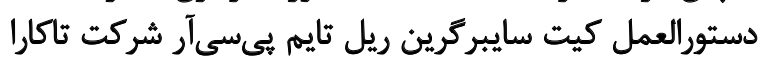
(Takara Bio Inc., Japan)

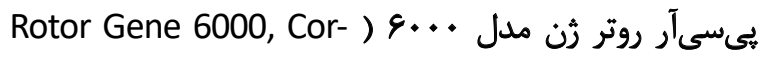
(bette, Korea

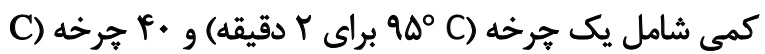

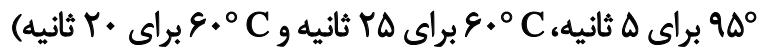

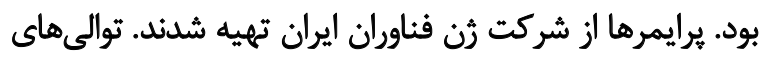

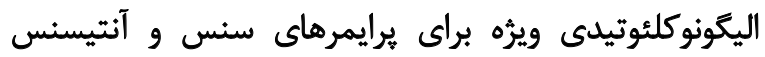

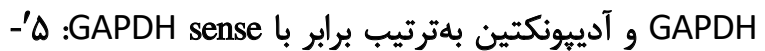
AAGTTCAACGGCACAGTCAAG -3' and GAPDH antisense: 5'- CATACTCAGCACCAGCATCAC - 3 '; adiponectin sense: $5^{\prime}$ - AATCCTGCCCAGTCATGAAG -3'and adiponectin antisense: 5' - CATCTCCTGGGTCACCCT-

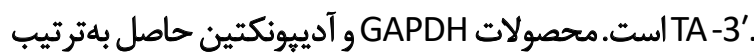

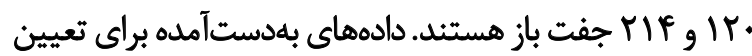

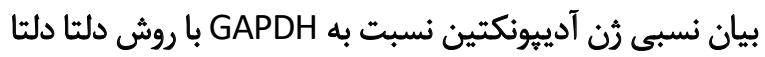
سي تى طبق فرمول بـ

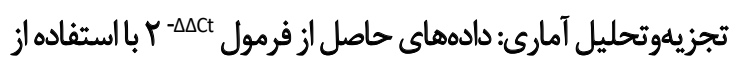

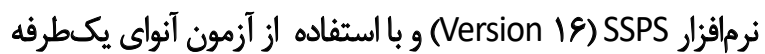

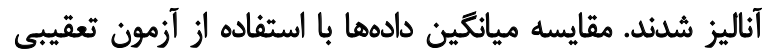

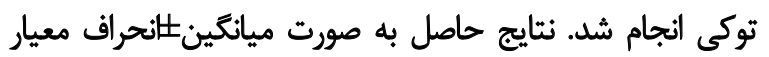

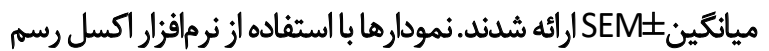

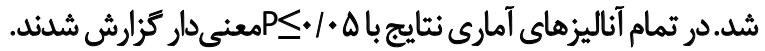

جدول ا. ميانكين غلظت سرمى هورمون لوتئينه(LH)

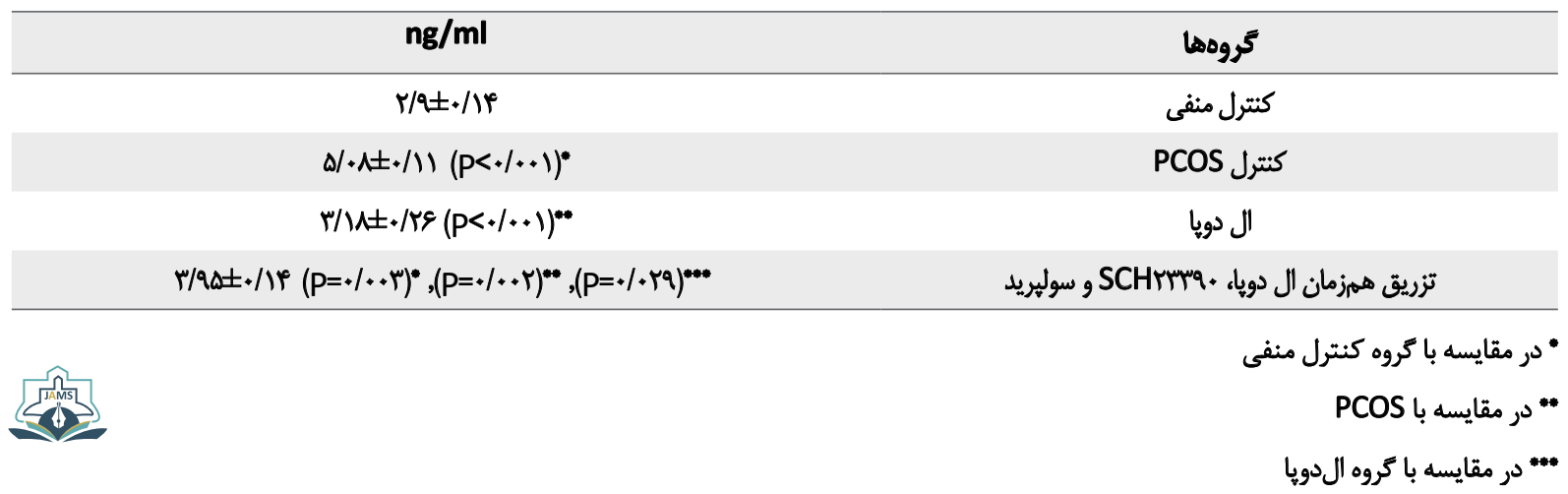




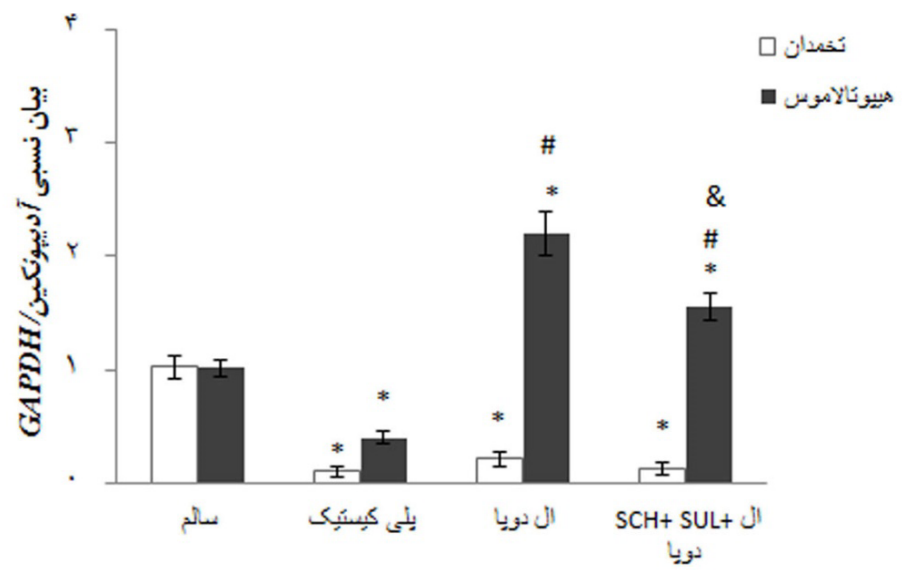

تصوير ا. ميانكين بيان نسبى رن آدييونكتين؛ :3: در مقايسه با سالم؛ \#: در مقايسه با PCOS؛ \&: در مقايسه با ال دويا

ميتواند سبب افزايش وزن و برمويى شود [عبك]. دويامين

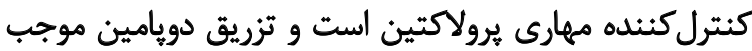

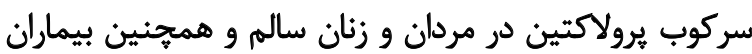

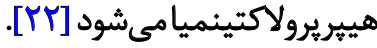

ازئز طرفى نشان داده شده است كه بين كاهش سطوح بيان

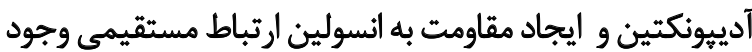

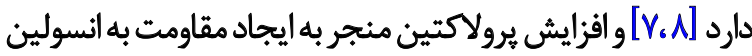

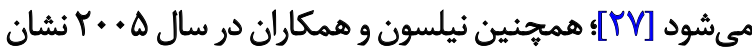

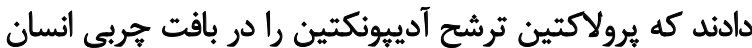

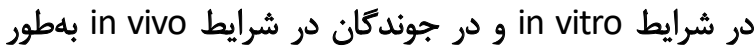

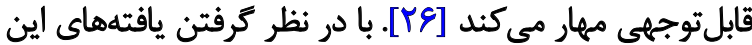

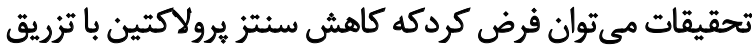

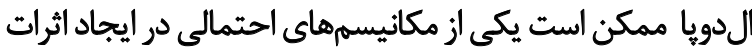

تحريكى دويامين بر بيان رن آدييونكتين در اين تحقيق بانيق باشد.

با در نظر كرفتن اثر مهارى دويامين بر آندرورثنهايي مثل

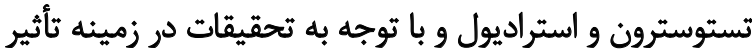

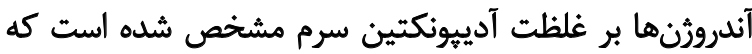

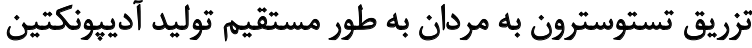

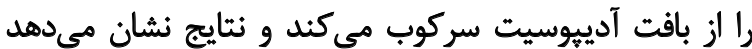

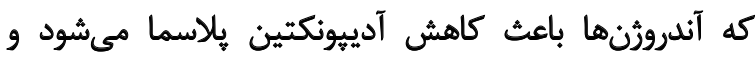

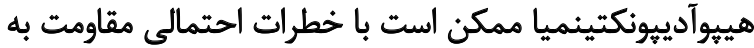

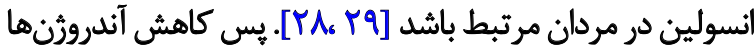

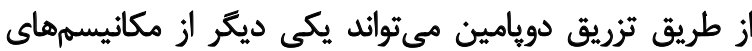

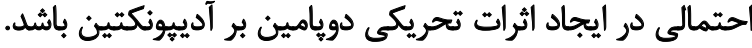

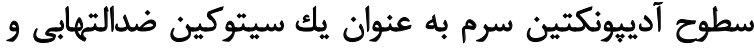

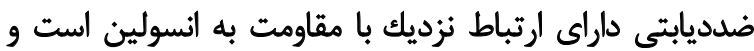

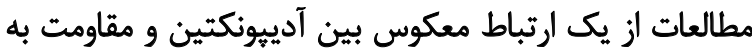

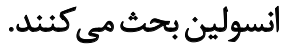

با توجه به اينكه مقاومت به انسولين يكى از مؤلفههاى

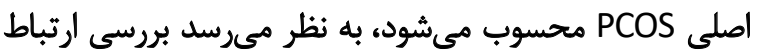

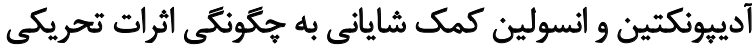

است كه هييرآندرورنيسم و جاقى در كاهش سطوح يلاسمايي

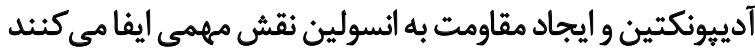

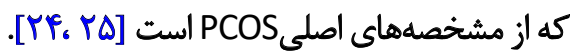
نتايج تحقيق حاضر نشان داد كه تزريق الدويا سبب افزيايش

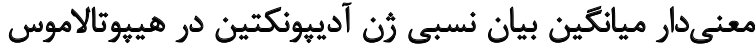

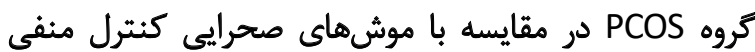

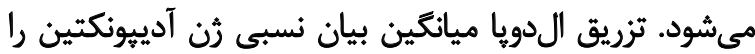

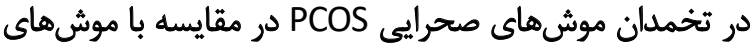

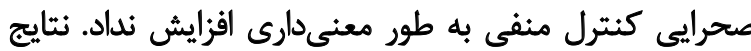

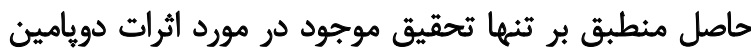

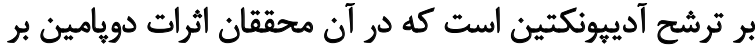

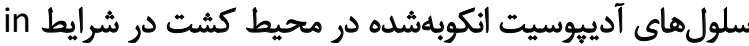

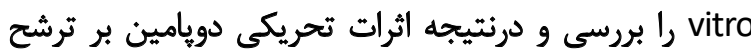

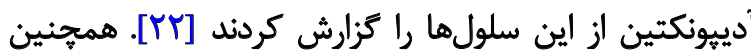

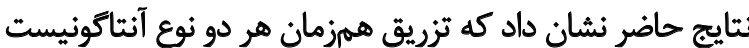

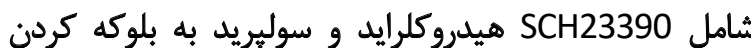

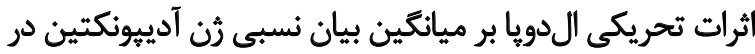

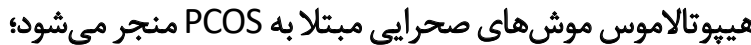

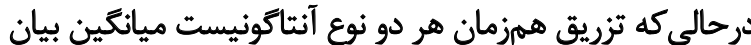

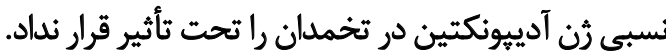

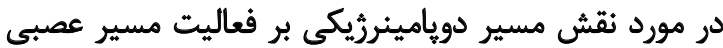

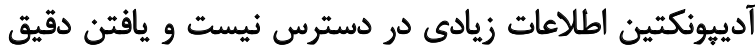

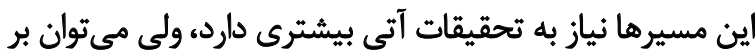

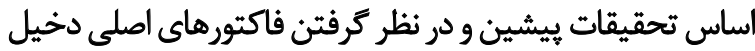

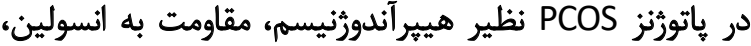

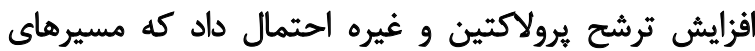

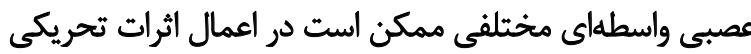

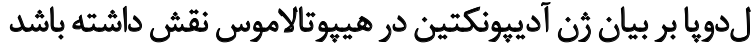

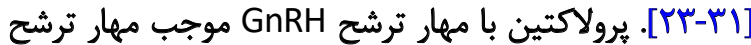

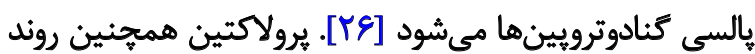

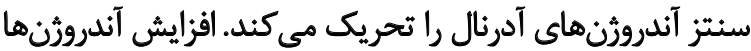


نتايج اين تحقيق مستخرج از إياننامه دانشجوى كارشناسى

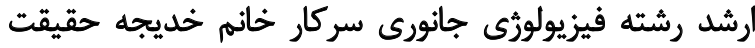

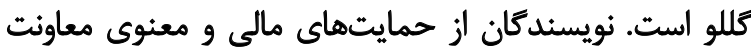

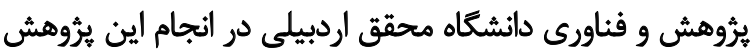

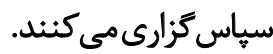

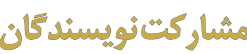

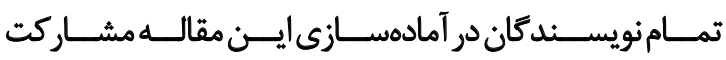

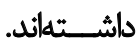

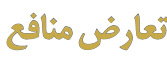

نويسندكان اعلام مى كنند كه هيجگُونه تضاد منافعى در اين

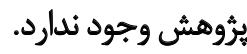

تشككر و قبدو هائي

نويسندكان از حمايت هاى مالى و معنوى معاونت برؤوهش و

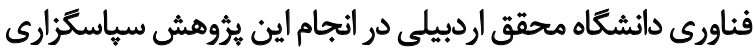

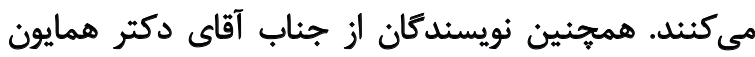

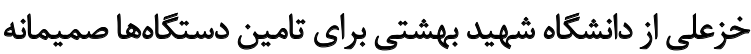

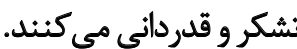

دويامين بر ترشح آدييونكتين در مدلهاى يلى كيستيك كند.

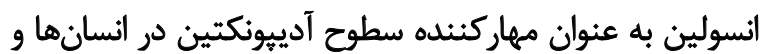

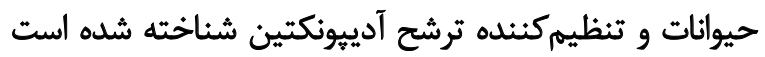

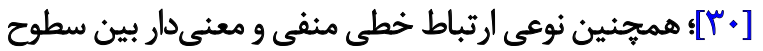

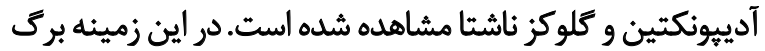

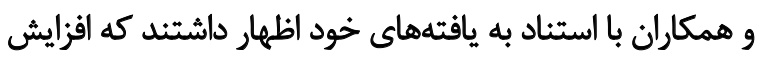

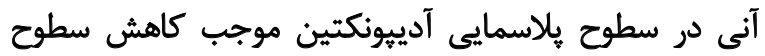

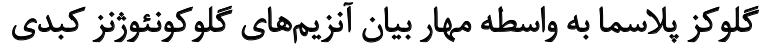

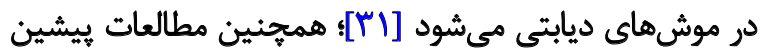

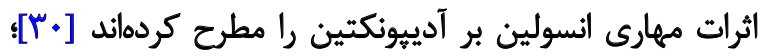

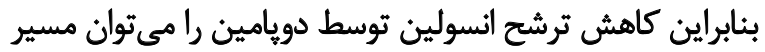

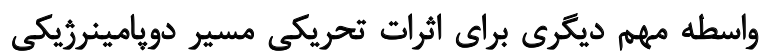

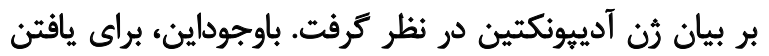

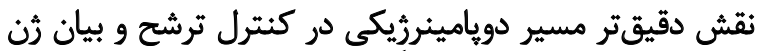

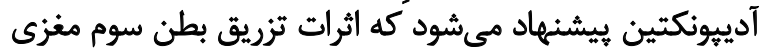

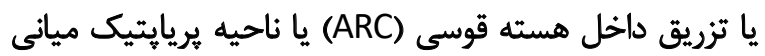

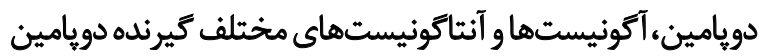

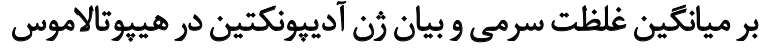

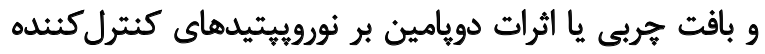

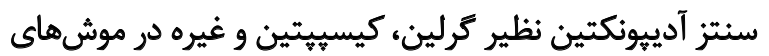
صحرايى PCOS بررسى شود. تثيجهيرى

القاى PCOSدر موش هاى صحرايي سبب افزايش غلظت سرمى

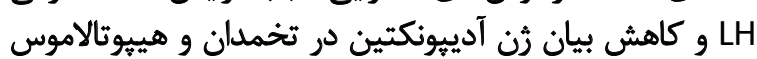

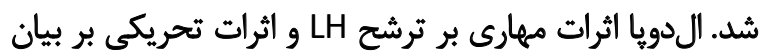

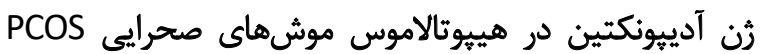

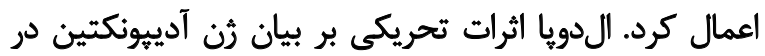

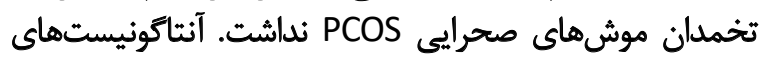

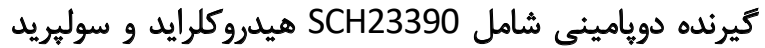

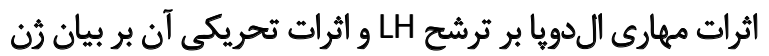

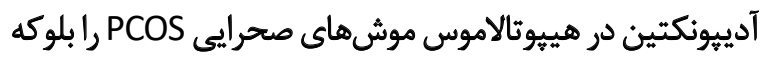

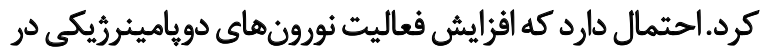

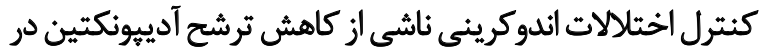
بيماران PCOS Pؤثر واقع شود.

ملاحظات اخلاقى

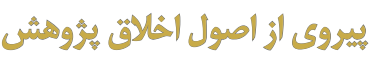

اين تحقيق راكميته يُروهشى دانشكاه محقق اردبيلى (كد: 1

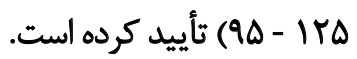




\section{References}

[1] Polak K, Czyzyk A, Simoncini T, Meczekalski B. New markers of insulin resistance in polycystic ovary syndrome. J Endocrinol Invest. 2017; 40(1):18. [DOI:10.1007/s40618-016-0523-8] [PMID] [PMCID]

[2] Adgi Z, Talaei A, Mohamadi Kelishadi M. [The evaluation of the relationship between hirsutism and insulin resistance in patients with PCOS and idiopathic hirsutism (Persian)]. J Arak Univ Med Sci. 2011; 14(2):51-7. http://jams.arakmu.ac.ir/article-1-953-en.html

[3] Dobrzyn K, Smolinska N, Kiezun M, Szeszko k, Rytelewska E, Kisielewska $\mathrm{K}$, et al. Adiponectin: A new regulator of female reproductive system. Int J Endocrinol. 2018; 2018:7965071. [DOI:10.1155/2018/7965071] [PMID] [PMCID]

[4] Davoodi B, Zilaei Bouri Sh, Ahangarpor A, Zilaei Bouri M. [Effects of two different physical exercises on plasma levels of adiponectin and resistin in obese and overweight young girls (Persian)]. J Arak Univ Med Sci. 2014; 17(4):27-37. http://jams.arakmu.ac.ir/article-1-2206-en.html

[5] Rodriguez-Pacheco F, Martinez-Fuentes AJ, Tovar S, Pinilla L, TenaSempere M, Dieguez C, et al. Regulation of pituitary cell function by adiponectin. Endocrinology. 2007; 148(1):401-10. [DOI:10.1210/ en.2006-1019] [PMID]

[6] Lee B, Shao J. Adiponectin and energy homeostasis. Rev Endocr Metab Disord. 2014; 15(2):149-56. [DOI:10.1007/s11154-013-9283-3] [PMID] [PMCID]

[7] Groth SW. Adiponectin and polycystic ovary syndrome. Biol Res Nurs. 2010; 12(1):62-72. [DOI:10.1177/1099800410371824] [PMID] [PMCID]

[8] Michalakis KG, Segars JH. The role of adiponectin in reproduction: From polycystic ovary syndrome to assisted reproduction. Fertil Steril. 2010; 94(6):1949-57. [DOI:10.1016/j.fertnstert.2010.05.010] [PMID] [PMCID]

[9] Cheng XB, Wen JP, Yang J, Yang Y, Ning G, Li XY. GnRH secretion is inhibited by adiponectin through activation of AMP-activated protein kinase and extracellular signal-regulated kinase. Endocrine. 2011; 39(1):6-12. [DOI:10.1007/s12020-010-9375-8] [PMID]

[10] Parillo F, Maranesi M, Mignini F, Marinelli L, Di Stefano A, Boiti C, et al. Evidence for a dopamine intrinsic direct role in the regulation of the ovary reproductive function: In vitro study on rabbit corpora lutea. PLoS One. 2014; 9(8):e104797. [DOI:10.1371/journal.pone.0104797] [PMID] [PMCID]

[11] Björklund A, Dunnett SB. Dopamine neuron systems in the brain An update. Trends Neurosci. 2007; 30(5):194-202. [DOI:10.1016/j. tins.2007.03.006] [PMID]

[12] Vallone D, Picetti R, Borrelli E. Structure and function of dopamine receptors. Neurosci Biobehav Rev. 2000; 24(1):125-32. [DOI:10.1016/ S0149-7634(99)00063-9]

[13] Fontaine R, Affaticati P, Yamamoto K, Jolly C, Bureau C, Baloche S, et al. Dopamine inhibits reproduction in female zebrafish (Danio rerio) via three pituitary D2 receptor subtypes. Endocrinology. 2013; 154(2):80718. [DOI:10.1210/en.2012-1759] [PMID]

[14] Zarabian M, Salehipour F, Ostad SN. The study of dose-response mitogenic effect of $\mathrm{L}$-dopa on the human periodontal ligament fibroblasts cell. Acta Med Iran. 2004; 42(5):363-6. https://acta.tums.ac.ir/index. php/acta/article/view/2752

[15] Liu X, Herbison AE. Dopamine regulation of gonadotropin-releasing hormone neuron excitability in male and female mice. Endocrinology. 2013; 154(1):340-50. [DOI:10.1210/en.2012-1602] [PMID]
[16] Venegas-Meneses B, Padilla JF, Juárez CE, Morán JL, Morán C, RosasMurrieta NH, et al. Effects of ovarian dopaminergic receptors on ovulation. Endocrine. 2015; 50(3):783-96. [DOI:10.1007/s12020-015-0636-4] [PMID]

[17] Chaudhari N, Dawalbhakta M, Nampoothiri L. GnRH dysregulation in Polycystic Ovarian Syndrome (PCOS) is a manifestation of an altered neurotransmitter profile. Reprod Biol Endocrinol. 2018; 16(1):37. [DOI:10.1186/s12958-018-0354-x] [PMID] [PMCID]

[18] Ayano G. Dopamine: Receptors, functions, synthesis, pathways, locations and mental disorders: Review of literatures. J Ment Disord Treat. 2016; 2(2):1000120. [DOI:10.4172/2471-271X.1000120]

[19] Gómez R, Ferrero H, Delgado-Rosas F, Gaytan M, Morales C, Zimmermann RC, et al. Evidences for the existence of a low dopaminergic tone in polycystic ovarian syndrome: Implications for OHSS development and treatment. J Clin Endocrinol Metab. 2011; 96(8):2484-92. [DOI:10.1210/ jc.2011-0075] [PMID]

[20] Andersson K, Fuxe K, Eneroth P, Härfstrand A, Agnati LF. Involvement of $D 1$ dopamine receptors in the nicotine-induced neuro-endocrine effects and depletion of diencephalic catecholamine stores in the male rat Neuroendocrinology. 1988; 48(2):188-200. [DOI:10.1159/000125007] [PMID]

[21] Grierson JP, James MD, Pearson JR, Wilson CA. The effect of selec tive D1 and D2 dopaminergic agents on sexual receptivity in the female rat. Neuropharmacology. 1988; 27(2):181-9. [DOI:10.1016/00283908(88)90169-4]

[22] Borcherding DC, Hugo ER, Idelman G, De Silva A, Richtand NW, Loftus J, et al. Dopamine receptors in human adipocytes: Expression and functions. PloS One. 2011; 6(9):e25537. [DOI:10.1371/journal. pone.0025537] [PMID] [PMCID]

[23] Lagaly DV, Aad PY, Grado-Ahuir JA, Hulsey LB, Spicer L. Role of adiponectin in regulating ovarian theca and granulosa cell function. Mol Cell Endocrinol. 2008; 284(1-2):38-45. [DOI:10.1016/j.mce.2008.01.007] [PMID]

[24] Escobar-Morreale HF, Villuendas G, Botella-Carretero Jl, Álvarez-Blasco F, Sanchón R, Luque-Ramírez M, et al. Adiponectin and resistin in PCOS A clinical, biochemical and molecular genetic study. Hum Reprod. 2006; 21(9):2257-65. [DOI:10.1093/humrep/del146] [PMID]

[25] Nishizawa H, Shimomura I, Kishida K, Maeda N, Kuriyama H, Nagaretani $\mathrm{H}$, et al. Androgens decrease plasma adiponectin, an insulinsensitizing adipocyte-derived protein. Diabetes. 2002; 51(9):2734-41 [DOI:10.2337/diabetes.51.9.2734] [PMID]

[26] Nilsson L, Binart N, Bohlooly-Y M, Bramnert M, Egecioglu E, Kindblom $\mathrm{J}$, et al. Prolactin and growth hormone regulate adiponectin secretion and receptor expression in adipose tissue. Biochem Biophys Res Commun. 2005; 331(4):1120-6. [DOI:10.1016/j.bbrc.2005.04.026] [PMID]

[27] Daimon M, Kamba A, Murakami H, Mizushiri S, Osonoi Sh, Yamaichi M, et al. Association between serum prolactin levels and insulin resistance in non-diabetic men. PLoS One. 2017; 12(4):e0175204. [DOI:10.1371/ journal.pone.0175204] [PMID] [PMCID]

[28] Xu A, Chan KW, Hoo RLC, Wang Y, Tan KCB, Zhang J, et al. Testosterone selectively reduces the high molecular weight form of adiponectin by inhibiting its secretion from adipocytes. J Biol Chem. 2005; 280(18):1807380. [DOI:10.1074/jbc.M414231200] [PMID]

[29] Page ST, Herbst KL, Amory JK, Coviello AD, Anawalt BD, Matsumoto $A M$, et al. Testosterone administration suppresses adiponectin levels in men. J Androl. 2005; 26(1):85-92. [DOI:10.1002/j.1939-4640.2005.tb02876.x] 
[30] Giahi L, Djazayery A, Rahimy A, Rahmany M, Larijani B. Serum level of adiponectin and its association with insulin sensitivity in overweight diabetic and non-diabetic Iranian men. Iran J Public Health. 2008; 37(2):8892. https://ijph.tums.ac.ir/index.php/ijph/article/view/2060

[31] Berg AH, Combs TP, Du X, Brownlee M, Scherer PE. The adipocyte-secreted protein Acrp30 enhances hepatic insulin action. Nat Med. 2001; 7(8):947-53. [DOI:10.1038/90992] [PMID] 